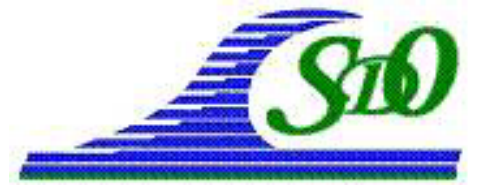

XI ${ }^{\text {èmes }}$ Journées Nationales Génie Côtier - Génie Civil

Les Sables d'Olonne, 22-25 juin 2010

DOI:10.5150/jngcgc.2010.021-B C Editions Paralia CFL

disponible en ligne - http://www.paralia.fr - available online

\title{
Caractérisation de la dynamique du lit de sable sous l'action des vagues
}

\author{
Céline BERNI ${ }^{1}$, Hervé MICHALLET ${ }^{1}$, Eric BARTHELEMY ${ }^{1}$
}

1. Laboratoire des Ecoulements Géophysiques et Industriels (UJF, GINP, CNRS), bp53, 38041 Grenoble cedex, France.

celine.berni@grenoble-inp.fr

\section{Résumé :}

Des mesures de profil de vitesses, de concentration et de la dynamique de la couche de fond sont analysées afin de mieux juger de l'importance relative des processus susceptibles de déstabiliser le lit d'une plage. Ces mesures ont été faites en laboratoire, dans un bassin de $30 \mathrm{~m} \times 30 \mathrm{~m}$, dans le cadre d'une expérience de morphodynamique de plage. Les effets de la contrainte de cisaillement, du gradient de pression, de l'accélération et de l'infiltration/exfiltration sont étudiés. Il ressort de cette étude que la contrainte de cisaillement est bien prépondérante, et qu'hormis cette contrainte, seuls les effets d'accélération pourraient jouer un rôle important.

\section{Mots-clés :}

Transport sédimentaire - Erosion - Sheet-flow (ruissellement en nappe) - Processus

\section{Introduction}

Le lit de sédiments qui constituent la plage est déstabilisé localement par l'action des vagues. Une fois mobilisé, le sédiment est transporté, ce qui conditionne l'évolution de la morphologie de la plage. Plusieurs processus peuvent être à l'origine d'une déstabilisation du lit. La contrainte de cisaillement de l'écoulement au fond est le principal moteur de cette déstabilisation, elle est généralement estimée grâce à la vitesse cross-shore hors de la couche limite (e.g. AUSTIN et al., 2009). Cependant, les modèles basés sur cette vitesse seulement se sont avérés insuffisants pour prédire le transport sédimentaire. NIELSEN (2006) a développé un modèle prenant aussi en compte les effets d'accélération associée aux fronts raides des vagues en zone de déferlement. Cette accélération permet de quantifier le gradient de pression horizontal qui s'exerce au fond sur les grains (FOSTER et al., 2006). D'autre part, TURNER \& MASSELINK (1998) ont développé un modèle prenant en compte les effets d'infiltration/exfiltration. L'infiltration engendre deux processus dont les effets sont contraires. Elle est accompagnée d'un gradient de pression vers le bas qui a tendance à stabiliser le lit; mais le flux vertical vers le fond contribue aussi à diminuer l'épaisseur de la couche limite par aspiration de celle-ci et ainsi à augmenter le potentiel de transport de l'écoulement. L'exfiltration offre un gradient de pression vers le haut qui déstabilise le sol mais dilate la couche limite, diminuant ainsi le potentiel de transport 
de l'écoulement. AUSTIN et al. (2009) ont comparé les deux modèles (avec accélération; avec infiltration/exfiltration) et tenté d'évaluer leurs importances respectives via un modèle de transport basé sur une équation de type Meyer-Peter Müller. Les résultats de leur étude montrent que les processus d'infiltration sont négligeables. Cependant, cette évaluation est indirecte, en effet, vitesse, accélération et concentration en sédiments sont souvent mesurées à une altitude fixe de la colonne d'eau et la dynamique de l'interface lit-fluide est mal connue. La présence notamment d'une fine couche mobile (d'épaisseur 10 à 60 diamètres de grain) fortement concentrée, que l'on appelle sheet-flow peut fortement affecter le transport sédimentaire et il est difficile d'en rendre compte via une modélisation de la contrainte au fond (HSU \& HANES, 2004).

On cherche ici à caractériser la réponse de l'interface lit-fluide et en particulier la sheetflow au passage des vagues grâce à des mesures au niveau de cette interface.

\section{Méthodes}

Une expérience de morphodynamique de plage a été réalisée en automne 2008 dans le bassin de génie côtier du LHF (Grenoble INP - Sogréah) de $30 \mathrm{~m}$ × 30 m équipé sur un bord d'un batteur "serpent" multi-éléments permettant de réaliser des houles aléatoires multi-directionnelles. Ces expériences ont été réalisées dans l'objectif de caractériser la dynamique d'un courant sagittal et les évolutions morphologiques associées (CASTELLE et al., 2010). Une plage de sable (de diamètre médian 166 microns) couvrait la largeur du bassin (30 m en longshore) pour un profil total de $19 \mathrm{~m}$ crossshore, sur une épaisseur d'environ 15 à $20 \mathrm{~cm}$ au-dessus d'un soubassement de gravier recouvert de géotextile. La pente moyenne de la plage est environ 1/25.

La houle générée présente un spectre de JONSWAP avec une hauteur significative de vague $H_{s}=18 \mathrm{~cm}$ en eau profonde ( $h=76,5 \mathrm{~cm}$ aux batteurs) et une période pic $T_{p}=3,5 \mathrm{~s}$. Les données présentées dans cet article sont relatives à un point situé à $15 \mathrm{~m}$ des batteurs et $3 \mathrm{~m}$ du bord du bassin, dans la zone de déferlement, pour $h=20 \mathrm{~cm}$. (figure 1a).

De nombreux instruments ont été déployés lors de cette expérience, cette étude ne porte que sur certains d'entre eux qui sont présentés sur la figure $1 b$.

Un vélocimètre acoustique (ADVP : Acoustic Doppler Velocity Profiler) mesure les 3 composantes de la vitesse instantanée (la composante cross-shore sera notée $u$, longshore $v$ et verticale $w$ ) tous les $3 \mathrm{~mm}$ sur un profil vertical. Les vitesses en chaque point du profil sont déduites de l'analyse des fréquences Doppler moyennées sur 32 tirs consécutifs (fréquence de répétition des tirs de $1600 \mathrm{~Hz}$, ce qui conduit à une résolution de 0,02 s ; cf. HURTHER, 2001, pour une description détaillée de l'appareil ; MIGNOT et al., 2009, pour des mesures en zone de déferlement). Les vitesses et l'accélération cross-shore du fluide hors de la couche limite (à $8 \mathrm{~cm}$ du fond) seront notées $u_{\infty}, w_{\infty}$ et $a_{\infty}$ (on vérifie que $v$ est très faible devant $u$ ). L'intensité du signal acoustique retrodiffusé 
en chaque point du profil vertical est également enregistrée. L'altitude du maximum d'intensité acoustique rétrodiffusée (moyennée sur 32 tirs consécutifs) donne la position d'une isopycne $(C \sim 210 \mathrm{~g} / \mathrm{L})$ correspondant à ce que l'on définit comme le sommet de la couche de sheet-flow. L'altitude du maximum de la différence entre la moyenne de l'intensité acoustique et sa variance (sur 32 tirs consécutifs) permet d'estimer la position du lit sous la couche de sheet-flow (BERNI et al., 2009; SILVA et al., in press). Le premier point de mesure au-dessus du lit ainsi défini fournit les vitesses verticale et cross-shore au fond, respectivement $w_{f}$ et $u_{f}$.

Cinq capteurs de mesure de pression interstitielle et huit capteurs optiques sont disposés verticalement de part et d'autre de l'interface sol-eau. Des mesures de pression est déduit le gradient de pression non-hydrostatique au travers de l'interface $P_{z}=\left(P_{5}-P_{3}-2 d\right) / d$, où $P_{5}$ et $P_{3}$ sont les pressions mesurées (converties en hauteur d'eau) par les capteurs 5 et 3 et $d$ la distance entre deux capteurs $(d=2 \mathrm{~cm}$; le lit étant situé approximativement au niveau de $P_{4}$ pour les mesures considérées).

Chaque capteur optique est constitué de deux fibres optiques, l'une émettrice et l'autre réceptrice. L'analyse de la lumière rétrodiffusée par les sédiments permet de caractériser la stabilité du lit et la présence de suspension à haute fréquence (environ $50 \mathrm{~Hz}$ ) sur un petit volume de mesure (quelques $\mathrm{mm}^{3}$ ), (BERNI et al., 2009). Des expériences ultérieures ont montré que, pour un substrat mobile, la valeur filtrée du signal (notée $C_{i}$ ) de la fibre $i$ est directement liée à la concentration. Pour cette étude, une calibration précise qui aurait permis la quantification de la concentration n'a pas pu être effectuée. La distinction entre substrat mobile et substrat stable se fait grâce à l'écart type du signal : des variations importantes du signal optique indiquent que le sédiment est mobile. Une sonde capacitive mesure les déplacements de la surface libre.

(a)

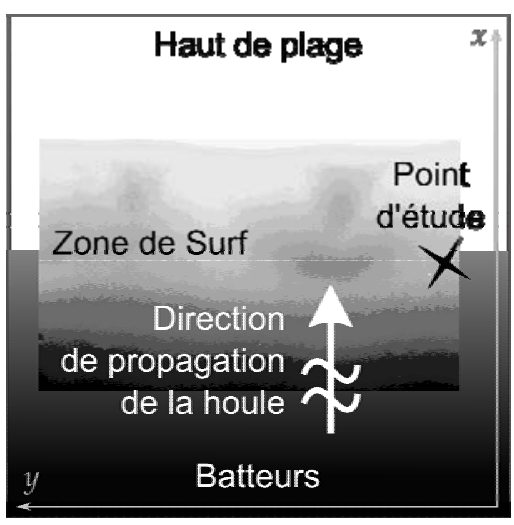

(b)

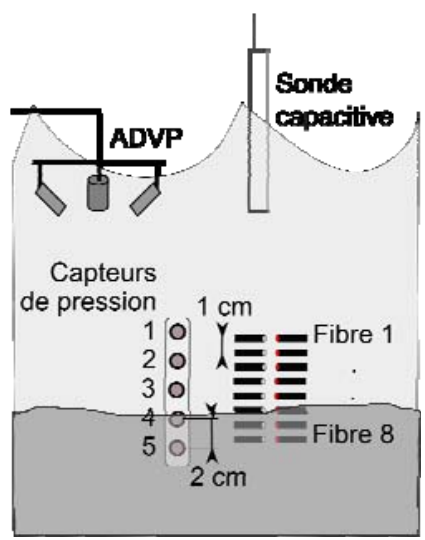

Figure 1. Schéma de l'installation : (a) vue en plan horizontal, sur la partie centrale figure la topographie de la plage (b) vue en plan vertical. 
(a)

(b)

(c)

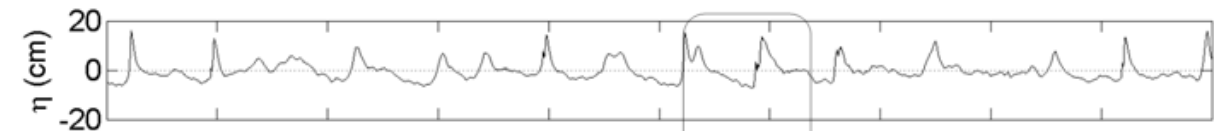

)

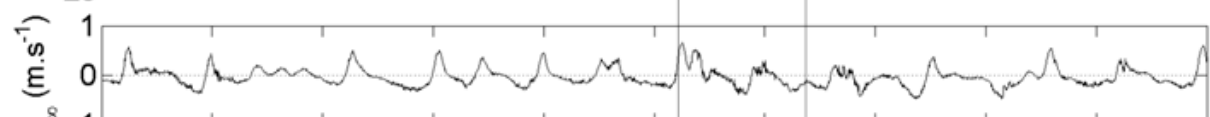

บ $^{8}$

-1
5
0

$\pi^{8}-5$

(d)

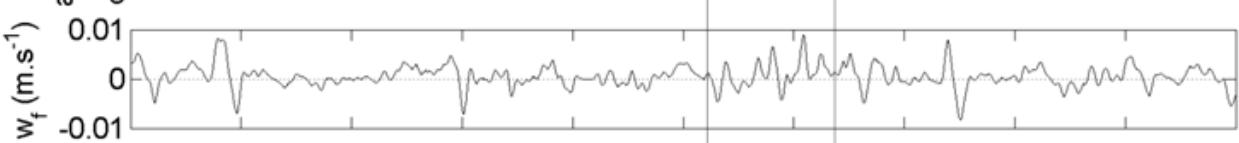

(e)

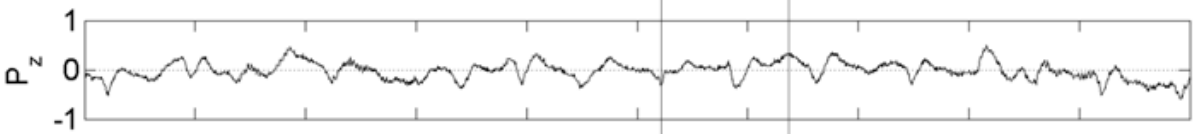

(f)

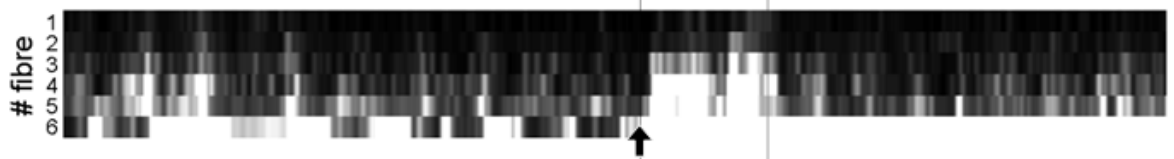

(g)

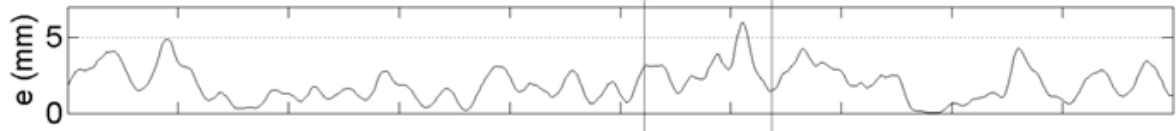

(h)

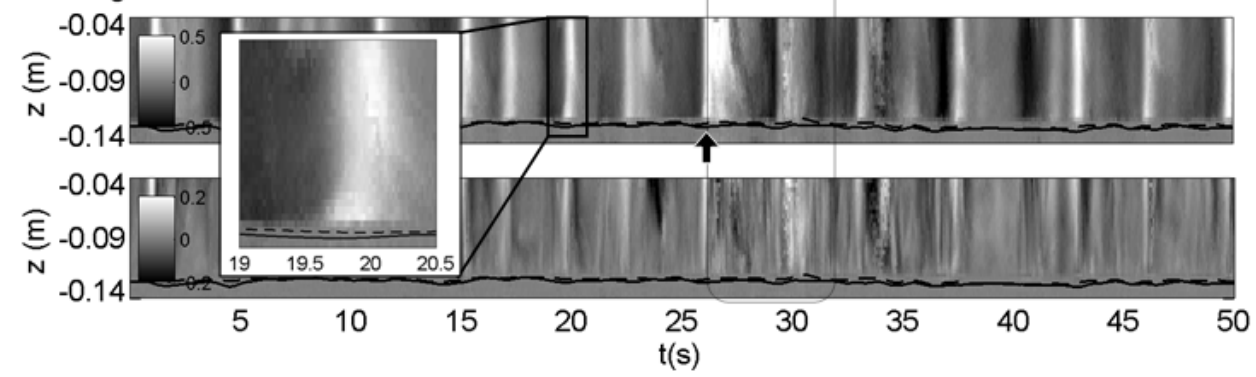

Figure 2. (a) Série temporelle de l'élévation de la surface libre;(b) vitesse cross-shore hors couche limite; (c) accélération cross-shore hors couche limite (déduite de la

dérivée temporelle de la vitesse); (d) vitesse verticale au fond; (e) gradient de pression $P_{z}$; (f) profil de concentration ; (g) épaisseur de sheet-flow e ; (h) profils des vitesses (en $\mathrm{m} / \mathrm{s}$ ) horizontale et (i) verticale sur lesquels sont tracées les positions $d u$ fond (trait plein) et du sommet de la sheet-flow. La distance z est donnée par rapport à la céramique émettrice de l'ADVP située environ $6 \mathrm{~cm}$ sous le niveau d'eau moyen.

\section{Résultats}

\subsection{Série temporelle à l'échelle de la vague}

Pour cette étude, une journée parmi les 50 jours de l'expérience a été sélectionnée. Au cours de cette journée, nous nous intéressons plus spécifiquement à un intervalle de 20 minutes dont une portion est montrée figure 2. L'accord entre déplacement de surface libre et vitesse hors couche limite est remarquable. Sont représentés conjointement le gradient de pression (e) et la vitesse verticale au fond (d) dont les variations présentent 
certaines similarités. Les profils verticaux de $u$ et $w$ sont représentés avec les niveaux du fond et du sommet de la sheet-flow figure $2 \mathrm{~h}$, i. L'avance de phase de $u$ dans la couche limite est visible, notamment à $\mathrm{t}=20 \mathrm{~s}$ ( $c f$. zoom). D'autre part, on observe un évènement remarquable à $t=26 \mathrm{~s}$. Une vague avec une crête secondaire engendre de fortes vitesses $u$ avec du déferlement, cause de fluctuations sur les profils de $u$ et $w$, et le développement d'une sheet-flow dépassant $5 \mathrm{~mm}$ d'épaisseur. Elle s'accompagne d'une mise en suspension notable du sable proche du fond, qui se solde par une accrétion de l'ordre de $1 \mathrm{~cm}$ enregistrée par les fibres optiques. (La couleur blanche de la figure $2 \mathrm{f}$ correspond à une forte concentration pouvant être synonyme de suspension fortement concentrée ou de fond. L'écart type du signal permet de les différencier.) Le nuage de suspension semble retomber un peu avant $t=29 \mathrm{~s}$ lorsque le gradient de pression et la vitesse verticale au fond deviennent brutalement négatifs. On retrouve le même schéma à $t=30 \mathrm{~s}$ quoique la retombée de sédiments semble précéder la chute de $P_{z}$ et $w_{f}$. L'observation de cette série temporelle suggère certains processus, l'étude des corrélations entre signaux va permettre de mieux les identifier.

\subsection{Corrélations}

AUSTIN et al. (2009) utilisent le gradient de pression pour estimer la vitesse verticale du fluide au fond grâce à la loi de Darcy qui les prédit proportionnels. Pour évaluer cette approche, une première étape consiste à s'assurer de la pertinence de la mesure de la vitesse au fond. La covariance entre $u_{\infty}$ et $w_{\infty}$ est nulle pour un déphasage nul (figure 3), Cette covariance présente un pic de 0,35 pour un retard de $0,3 \mathrm{~s}$ de $w_{\infty}$ par rapport à $u_{\infty}$ et son symétrique $(-0,3$ à $-0,3 \mathrm{~s})$. Ces déphasages relativement faibles traduisent le fort pincement des vagues en zone de déferlement. Les vitesses $u_{\infty}$ et $w_{f}$ sont anti-corrélées $(0,35)$ avec une légère avance de $w_{f}$ sur $u_{\infty}(0,1 \mathrm{~s})$. Ces différences suggèrent que la vitesse mesurée au fond n'est pas un simple résidu de la vitesse hors couche limite. La corrélation entre $u_{\infty}$ et $P_{z}$ présente une forme similaire à celle calculée par AUSTIN et al. $\left(2009\right.$, Fig. $\left.7: \mathrm{r}_{\mathrm{u}, \mathrm{w}}\right)$ i.e. une corrélation nulle en $-0,1 \mathrm{~s}$, assortie d'un pic négatif $(-0,3)$ pour un déphasage d'environ $0,25 \mathrm{~s}$. Ainsi, $w_{f}$ et $P_{z}$ sont tous deux anti-corrélés à $u_{\infty}$. Ceci semble valider l'estimation de la vitesse verticale au fond par mesure directe. Cependant $P_{z}$ est en avance d'un peu plus d'un dixième de seconde sur $w_{f}$, et la corrélation $\left[P_{z}, w_{f}\right]$ est fortement asymétrique. D'autre part, les pics secondaires des covariances $\left[u_{\infty}, P_{z}\right]$ et $\left[u_{\infty}, w_{f}\right]$ sont décalés. Ainsi l'amalgame gradient de pression et vitesse verticale au fond n'est pas évident.

Les covariances entre l'épaisseur de sheet-flow e et $u_{\infty}, a_{\infty}, w_{f}$ et $P_{z}$ sont représentées sur la figure 4. L'épaisseur de sheet-flow est en phase avec $u_{\infty}$ et $u_{f}$, et en retard sur $w_{f}$ de plus d'une seconde. D'autre part, la corrélation entre $e$ et $w_{f}$ est négative pour un déphasage nul. Ceci suggère que l'épaisseur de sheet-flow est maximale pour une vitesse $u>0$ vers la côte et une infiltration dans le sol $(w<0)$. Par ailleurs, la covariance entre $P_{z}$ et $e$ est la plus marquée $(0,27)$ et donne une épaisseur de sheet-flow maximale 
en avance de phase de 0,5 s par rapport au gradient de pression. Ceci semble indiquer que le gradient de pression n'est pas moteur dans la formation de la couche de sheetflow. Quant au gradient de pression horizontal, déduit de $a_{\infty}$, il est corrélé à $e$, avec une avance de 0,5 s. Ainsi, un pic d'accélération est suivi d'un pic de concentration. On retrouve les résultats d'AUSTIN et al. (2009) qui tendent à suggérer que l'accélération est moteur dans la déstabilisation du lit.
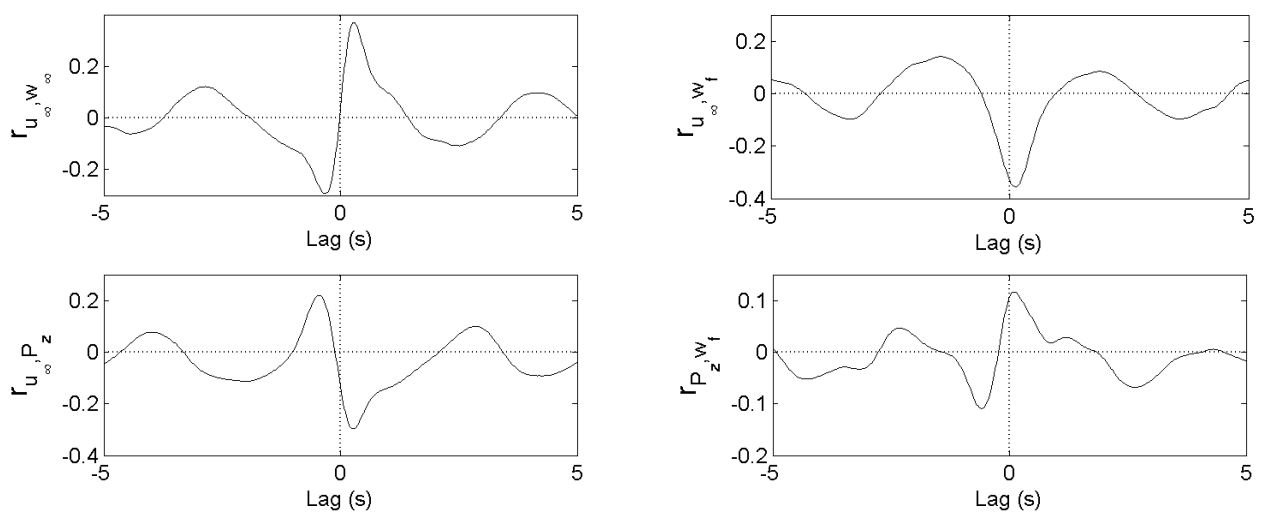

Figure 3. Covariances de $u_{\infty}$ avec $w_{\infty}$, avec $w_{f}$ et avec $P_{z}$, et covariance de $w_{f}$ avec $P_{z}$ calculées sur 20 minutes.
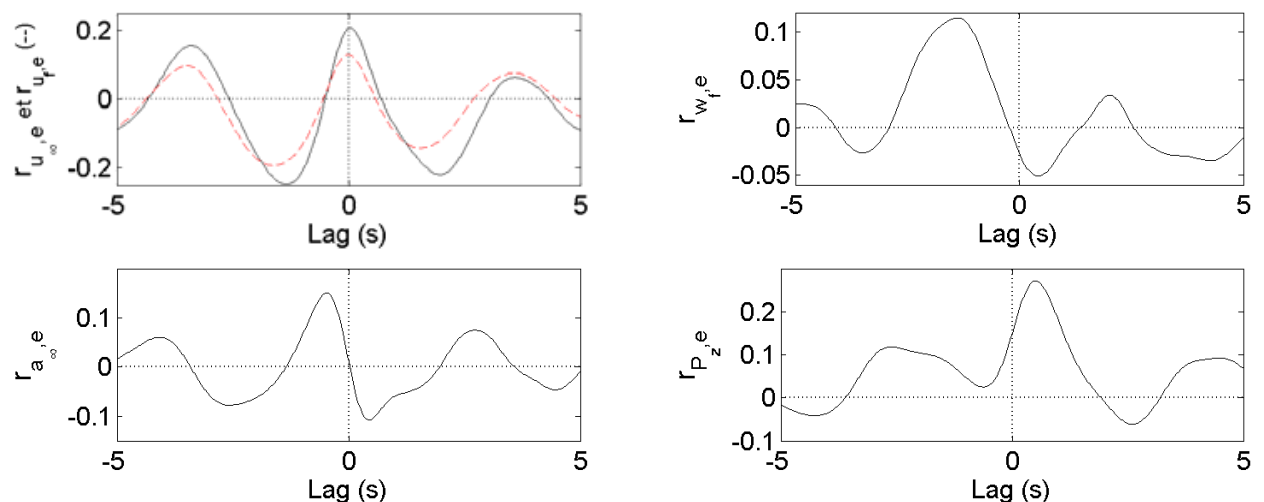

Figure 4. Corrélations avec l'épaisseur de sheet-flow e, de $u_{\infty}, a_{\infty},(u w)_{f}, w_{f}, P_{z}$.

Des corrélations avec les signaux de fibres optiques sont présentées figure 5. La concentration proche du fond (fibre 6) est presque en phase avec $u_{\infty}(<0,2 \mathrm{~s}$ de retard) comme l'était $e$, puis en montant dans la colonne d'eau, la concentration prend du retard vis-à-vis de $u_{\infty}$ (figure $5 \mathrm{a}$ ) et devient de moins en moins nette. Ceci est en adéquation avec les résultats de DOHMEN-JANSSEN \& HANES (2005). Le nuage de suspension lié à la dynamique de la couche limite se développe au fond, puis monte dans la colonne d'eau. Afin de mieux comprendre ce qui initie cette mise en suspension, nous avons corrélé le gradient de pression, la vitesse verticale au fond et l'accélération à $C_{6}$ (figure 5b). Le gradient de pression est plutôt bien corrélé en 0 avec la concentration $C_{6}$ tandis 
que la corrélation $\left[w_{f}, C_{6}\right]$ est médiocre. La corrélation entre l'accélération et la concentration $C_{6}$ est plutôt bonne avec une légère avance de phase. Ces observations semblent confirmer que l'exfiltration ne joue qu'un rôle mineur dans la déstabilisation du lit.

(a)
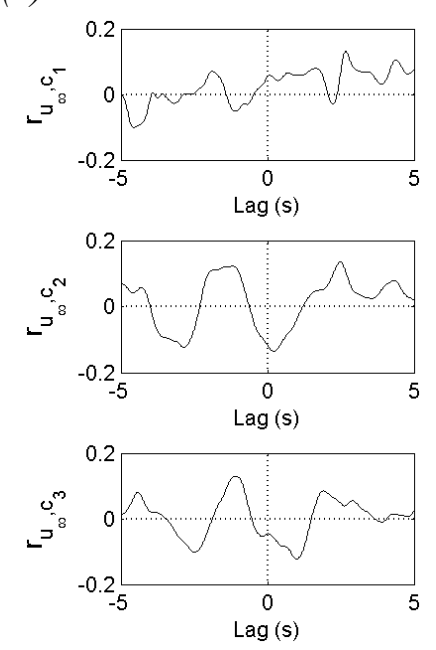

(b)
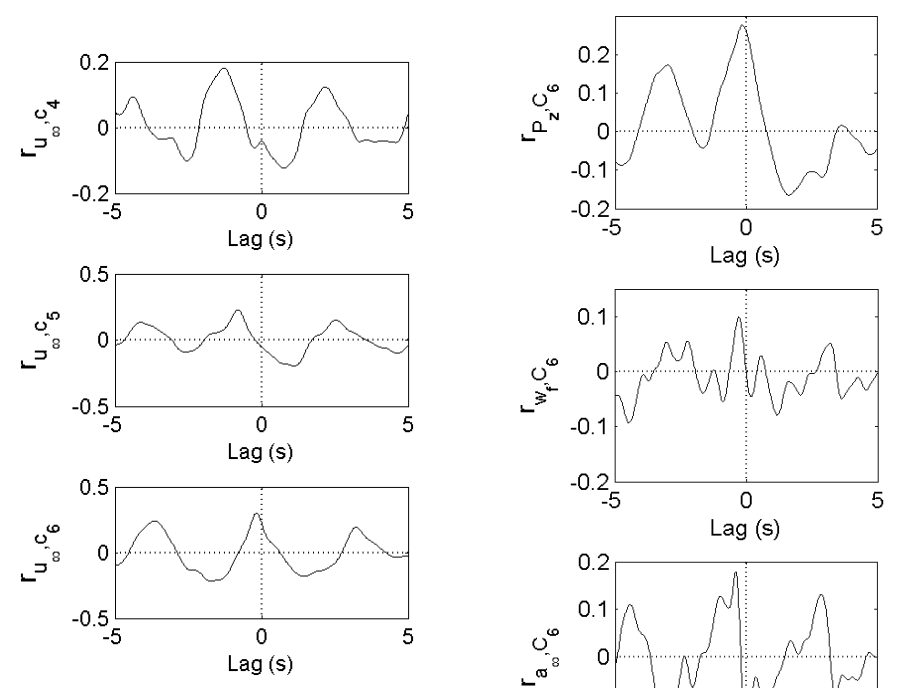
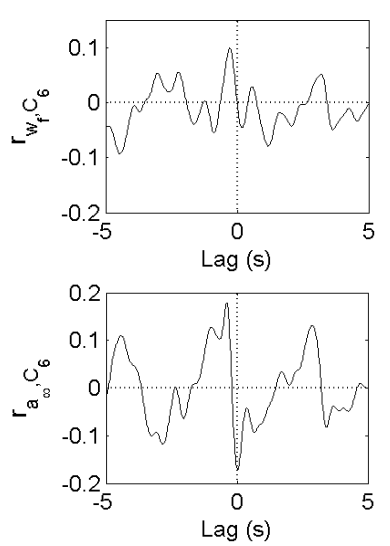

Figure 5. Corrélations de $u_{\infty}$ avec $C_{i} i$ désignant l'une des 6 fibres dans la colonne d'eau, calculées sur 100 s pendant lesquelles la fibre 6 n'est pas dans le sable.

\section{Conclusion}

La loi de Darcy prédisant une vitesse verticale proportionnelle au gradient de pression à travers l'interface ne rend pas compte de nos expériences. Ceci peut être dû en partie au fait qu'un des capteurs de pression était dans la colonne d'eau, soumis à une pression dynamique plus forte. De nouvelles expériences doivent être menées pour comparer le gradient de pression entre 2 capteurs dans le sol et la vitesse verticale au fond mesurée grâce au profileur acoustique.

D'autre part, cette étude conduit à penser que la contrainte au fond, que l'on peut estimer proportionnelle à $u_{\infty}{ }^{2}$, joue un rôle essentiel dans la déstabilisation du lit. Ce rôle pourrait être amplifié par des vitesses d'infiltration qui réduiraient l'épaisseur de la couche limite mais ce dernier point reste à confirmer. Le rôle de l'accélération dans cette déstabilisation reste incertain. Elle pourrait être un initiateur. Une étude vague à vague qui mettrait en parallèle accélération maximale et concentration moyenne sur la vague permettrait peut-être d'éclaircir ce point.

Enfin, la dynamique de la sheet-flow est en phase et fortement corrélée à la vitesse cross-shore hors de la couche limite, tandis que le nuage de suspension met 1 à $2 \mathrm{~s}$ pour se former et monter dans la colonne d'eau. 
Thème 2 -Dynamique sédimentaire et transports des particules

Remerciements : Cette étude, menée dans le bassin du LHF (INPG - SOGREAH), a été partiellement financée par l'ANR COPTER et le projet MODLIT (DGA/SHOM INSU/RELIEFS). Le soutien technique de SOGREAH, en particulier G. Excoffier et L. Marcellin, a été fortement apprécié. Nous souhaitons aussi remercier tous les participants, ponctuels ou réguliers, à cette grande expérience.

\section{Références bibliographiques}

AUSTIN M., MASSELINK G., O'HARE T., RUSSELL P. (2009). Onshore sediment transport on a sandy beach under varied wave conditions: Flow velocity skewness, wave asymmetry or bed ventilation? Marine Geology, 259, pp 86-101. doi:10.1016/j.margeo.2009.01.001

BERNI C., MIGNOT E., MICHALlET H., DALlA-COSTA C., GRASSO F., LAGAUZERE M. (2009). Diversity of bed evolution at wave and tidal scales on TrucVert beach. J. of Coast. Res., SI 56, pp 1726-1730.

CASTElle B., MiCHALlet H., MARIEU V., LECKLER F., DUBARDIER B., LAMBERT A., BERNI C., BARTHELEMY E., BOUCHETTE F., BONNETON P., KIMMOUN O., SOUS D., ALMAR R. (2010). Modélisation physique des courants d'arrachement : apport des mesures lagrangiennes, XI ${ }^{\text {èmes }}$ Journées Nationales Génie Côtier- Génie Civil, Les Sables d'Olonne, pp 53-60. doi:10.5150/jngcgc.2010.007-C

DOHMEN-JANSSEN C.M., HANES D.M. (2005). Sheet flow and suspended sediment due to wave groups in a large wave flume. J. of Continental Shelf Res., 25, pp 333-347. doi:10.1016/j.csr.2004.10.009

FOSTER D.L. BOWEN A.J., HOLMAN R.A., NATOO P. (2006). Field evidence of pressure gradient induced incipient motion. J. of Geophysical Res., 11, C05004. doi:10.1029/2004JC002863

HSU T.-J., HANES D.M. (2004). Effects of wave shape on sheet flow sediment transport, J. Geophys. Res., 109, C05025. doi:10.1029/2003JC002075

HURTHER D. (2001). 3-D acoustic Doppler velocimetry and turbulence in openchannel flow. Thèse, Ecole Polytechnique Fédérale de Lausanne.

MIGNOT E., HURTHER D., CHASSAGNEUX F.-X., BARNOUD J.-M. (2009). A field study of the ripple vortex shedding process in the shoaling zone of a macro-tidal sandy beach. J. Coast. Res., SI 56, pp 1776-1780.

NIELSEN P. (2006). Sheet flow sediment transport under waves with acceleration skewness and boundary layer streaming. Coastal Engineering, 53, pp 749-758. doi:10.1016/j.coastaleng.2006.03.006

SILVA P.A., ABREU T., MICHALlET H., HURTHER D., SANCHO F. (in press). Sheet flow layer structure under oscillatory flows, Proc. River, Coastal and Estuarine Morphodynamics: RCEM 2009.

TURNER I.L., MASSELINK G. (1998). Swash infiltration-exfiltration and sediment transport. J. Geophys. Res. 103, pp 30813-30824. doi:10.1029/98JC02606 